\title{
ESTUDO DO DESEQUILÍBRIO DA FORÇA MÁXIMA ENTRE OS GRUPOS MUSCULARES ROTADORES MEDIAIS E ROTADORES LATERAIS DA ARTICULAÇÃO DO OMBRO, EM JOGADORES DA CATEGORIA JUVENIL DE BEISEBOL
}

\author{
Ana Cristina de Sousa * \\ Giany Gonze Tellini ** \\ Dr. Sérgio José Nicoletti ***
}

*Mestre em Ciências da Saúde - Área de Reabilitação do Departamento de Ortopedia e Traumatologia da UNIFESP. Escola Paulista de Medicina

** Doutora em Ciências da Saúde- Área de Reabilitação e Coordenadora do Curso de Especialização em Fisioterapia Motora Hospitalar e Ambulatorial da Disciplina de Fisiatria do Departamento de Ortopedia e Traumatologia da UNIFESP- Escola Paulista de Medicina *** Doutor em Medicina pela UNIFESP/EPM.

Trabalho realizado na Disciplina de Fisiatria e Grupo de Cirurgia de Ombro e Cotovelo da Disciplina de Cirurgia de Mão e Membro Superior do Departamento de Ortopedia e Traumatologia da UNIFESP-EPM ( Serviço do Prof. Dr. José Laredo Filho ), para obtenção do título de mestre em ciências da saúde.

Endereço para correspondência:

Rua Mario Barreto,66

CEP: 09041-390 Santo André - SP

Tel:(011)28666887. 
RESUMO : Estudo do desequilíbrio da força muscular entre rotadores mediais e laterais durante 0 arremesso na prática de beisebol em 40 atletas juvenis de nível competitivo. Os atletas foram divididos em dois grupos, sendo o grupo de estudo constituído de 14 atletas arremessadores e 0 grupo controle de 26 atletas não arremessadores. A força muscular destes atletas foi avaliada por meio de dinamometria isométrica de mola, em duas posições (abdução à 90 graus e posição neutra) tanto para 0 ato de arremesso quanto para outros gestos decorrentes da prática deste esporte. 0 objetivo deste estudo foi 0 de estabelecer possível relação do desequilíbrio da força muscular dos musculos rotadores mediais e laterais durante 0 arremesso e correlacionar a instabilidade da articulação glenumeral quanto ao lado dominante e não dominante do atleta. Os resultados mostraram uma diferença estatisticamente significante na avaliação do desequilíbrio da força muscular em posição neutra e abdução à 90 graus no lado não dominante .

ABSTRACT : Study about the unbalances muscle strength between the internal and external rotator muscles of the shoulder in 40 baseball pitchers. The athletes were divided in two groups. The study group consisted of 14 pitchers and the control group of 26 non-pitchers. Their muscle strength was evalueted by isometric dynamometry in two positions (abduction at 90 degrees and neutral) in the act of throwing and other positions derived from the gestures required in this game. The study estabilish the relation of unbalances muscles strength of the internal and external rotator muscles during the practice of pitching and instabilite in the dominating and non-dominating shoulder.The results showed statistically significant differences in the evaluation of unbalances muscle strength in neutral position and abduction at 90 degrees in the non-dominating shoulder.

\section{INTRODUÇÃO:}

O arremessador é um atleta que desempenha um papel fundamental no jogo de beisebol. Seu objetivo é arremessar a bola contra a posição do adversário com a maior quantidade de energia cinética possível. Para conseguir tais resultados, o arremessador precisa desenvolver habilidades que 0 capacitem a imprimir grande aceleração à bola, de maneira que a mesma alcance velocidades de $86,9 \mathrm{~m} / \mathrm{seg}^{9}$

Ao mesmo tempo em que imprime aceleração linear à bola, o arremessador usa a musculatura do membro superior para criar movimento circular, de maneira a produzir o chamado efeito "spin", cuja obtenção requer esforço adicional significativo, considerado impróprio para crianças e adolescentes que praticam o beisebol. ${ }^{14}$

A organização da prática esportiva impõe regras e estabelece deveres que acarretam esforços físicos padronizados, destinados a desenvolver as habilidades e o padrão neuromotor, necessário para a execução eficaz dos gestos desportivos, que compõem a modalidade praticada. Tal situação tende a produzir desequilíbrios funcionais, como o predomínio da força de grupos musculares mais usados para a realização dos movimentos mais freqüentes de um determinado gesto esportivo, como é o caso do arremesso do beisebol. ${ }^{3}$

Hoje o que se aplica no treinamento de atletas, do esporte competitivo profissional ou amador, é a teoria de automatização $0^{12}$ que tem como objetivo desenvolver no atleta, a capacidade de dominar com segurança a execução do movimento, atingindo o que conhecemos como estereótipo dinâmico motriz. ${ }^{16}$ Para que este resultado seja alcançado é necessário que 0 mesmo movimento seja 
repetido inúmeras vezes, até que se atinja a automatização. O número excessivo de repetições gestuais, realizadas com grande intensidade, provoca a maioria dos problemas de saúde associados à prática do esporte e comprometem a carreira do atleta. ${ }^{11}$

Presume-se, que o padrão de utilização do ombro dos jogadores profissionais e amadores de Beisebol, acaba por determinar o aumento da amplitude dos movimentos de rotação externa. Esse aumento do limite de amplitude do arco de movimento se deve, teoricamente, à deformação física da cápsula articular glenoumeral do ombro dominante, decorrente das cargas excepcionalmente elevadas, que durante certas fases do lançamento, a cabeça do úmero imprime sobre o complexo capsuloligamentar glenoumeral, causando "lasseamento" do tecido conjuntivo e aumento do volume da "câmara" articular.,214

Com base nessas informações, motivamo-nos a analisar comparativamente o desequilíbrio da força muscular entre rotadores mediais e laterais durante 0 arremesso na prática do beisebol, procurando estabelecer uma correlação da instabilidade glenumeral quanto ao lado dominante e não dominante do atleta.

\section{MATERIAL E MÉTODO:}

Neste estudo são analisados 40 atletas do sexo masculino, oriundos de diferentes estados brasileiros, que faziam parte dos quadros dos clubes, que disputaram vagas na seleção brasileira juvenil de beisebol, para os jogos amistosos no Japão, em 1998.

O estudo foi realizado no período entre 1998 a 2000, na Disciplina de Fisiatria e no Grupo de Cirurgia do Ombro e Cotovelo, na Disciplina da Mão e Membro Superior, do Departamento de Ortopedia e Traumatologia da UNIFESP- Escola Paulista de Medicina. Os dados foram colhidos durante os meses de janeiro a março de 1998, no Clube de Campo Bunkiô, da colônia japonesa, em Mogi das Cruzes, interior de São Paulo, durante os períodos de concentração dos jogadores para as provas seletivas. A idade dos atletas variou de 15 a 18 com média de 16,9 anos. 0 tempo de prática do esporte variou de 4 a 13 anos, com média de 9,8 anos. Os critérios de inclusão para o estudo foram os seguintes: atletas do sexo masculino com idades entre 16 e 18 anos e tempo mínimo de treinamento de dois anos em uma só posição, com interrupção máxima de um mês por ano. E os critérios de exclusão foram: presença de dor, durante o teste de força máxima, ter se submetido a intervenção cirúrgica em membros superiores, ter diagnóstico prévio de patologia de ombro e ter realizado tratamento conservador para tal e ser ambidestro.

Os atletas foram divididos em dois Grupo AR, ou Grupo de Estudo, onde foram selecionados apenas os arremessadores, dos quais 14 exerciam essa função e Grupo NAR ou Grupo Controle, sendo 26 considerados como não arremessadores.

Para avaliação da força muscular foi utilizado um dinamômetro de mola da marca CROWN ${ }^{\circledR}$, carga máxima de 30 kilogramas, fixado em um suporte metálico. Para tal avaliação, os atletas foram avaliados em duas posições: 1) sentado: os atletas foram posicionados com os membros inferiores fletidos à 90 graus de acetábulo-femoral e joelhos com os pés fixos no solo. Os membros superiores foram apoiados em uma mesa na posição de 90 graus de abdução e rotação neutra da articulação gleno-umeral e 90 graus da articulação cubital. Foram realizadas três medidas de força para cada grupo muscular (medial e lateral), bilateralmente, com intervalo de tempo de 60 segundos entre uma medida. Realizava-se o teste uma vez em um membro alterando com o outro membro e 2) em pé: a força dos rotadores medial e lateral de ombro na posição em pé com o membro superior em posição neutra, utilizando o dinamômetro de mola com fixação. Os atletas foram posicionados com os 
membros inferiores em abdução funcional da articulação acetábulo-femoral e $30^{\circ}$. de flexão de joelhos com os pés fixos no solo. Os membros superiores foram apoiados próximos ao corpo e rotação neutra da articulação gleno-umeral e 90 graus da articulação cubital. Foram realizadas três medidas de força para cada grupo muscular (medial e lateral), bilateralmente, com intervalo de tempo de 60 segundos entre uma medida. Realizava-se o teste uma vez em um membro alterando com 0 outro membro. As variáveis estudadas foram força máxima de rotação medial e lateral dos ombros dominantes e não dominantes, presença de sinal do sulco e tempo de treinamento. Esta coleta de dados de força muscular máxima foi realizada por uma equipe formada por três fisioterapeutas, coordenados e treinados pela autora deste trabalho. Para a análise estatística utilizamos os Testes de Mann-Whitney, Qui-Quadrado, Exato de Fisher e McNemar. ${ }^{18}$

\section{RESULTADOS:}

Os resultados encontrados neste estudo foram o equilíbrio de força máxima entre os grupos musculares de rotadores mediais e laterais no ombro dominante esteve presente em 5 atletas (12,5\%), sendo 2 atletas arremessadores (5\%) e 3 atletas não arremessadores (7,5\%). Houve predomínio de rotadores laterais em 4 arremessadores (10,0\%) e 9 não arremessadores (22,5\%). A rotação medial teve predomínio nos ombros de 8 atletas arremessadores (20\%) e 14 não arremessadores $(35,0 \%)$. Dos atletas que apresentaram desequilíbrio de força máxima, 12 arremessadores $(34,3 \%)$, o ombro dominante apresentou predomínio dos músculos rotadores mediais em $8(66,7 \%)$ dos atletas. Em 4 (33,3\%) atletas arremessadores, o ombro dominante apresentou predomínio dos rotadores laterais. Dentre os 23 não arremessadores com desequilíbrio de força máxima $(65,7 \%)$, o ombro dominante apresentou predomínio dos músculos rotadores mediais em $14(60,9 \%)$ e dos rotadores laterais, em 9 (39,1\%). No grupo não arremessadores, 11 atletas $(64,7 \%)$ apresentaram predomínio de rotadores laterais e 6 (35,2\%), de rotadores mediais. Foram encontradas diferenças estatisticamente significantes para 0 predomínio de rotadores laterais, em posição neutra nos não arremessadores. Foram encontradas diferenças estatisticamente significantes $(P<0,001)$ entre o predomínio de força máxima entre rotadores mediais e laterais em abdução de $90^{\circ}$. em arremessadores e não arremessadores. Os valores obtidos em arremessadores e não arremessadores foram estatisticamente significantes $(P=0,001)$, nesta distribuição, encontramos 9 arremessadores $(69,2 \%)$ com predomínio de rotadores laterais e 4 $(30,7 \%)$ de mediais. No grupo não arremessadores, 7 (35,0\%) apresentaram predomínio de rotadores laterais e $13(65,0 \%)$ de mediais.

\section{DISCUSSÃO:}

O estudo comparativo do desequilíbrio de força máxima entre os músculos rotadores mediais e os músculos rotadores laterais do ombro em jogadores de beisebol, traduz uma preocupação que vem sendo apresentada por vários autores, que é a prevalência de dor nos ombros dos atletas cujo gesto esportivo principal é o lançamento. Vários desses autores atribuem ao desequilíbrio de forças entre os grupos musculares, que controlam os movimentos do ombro. Dentre as diferentes fases de movimentos executados acima da cabeça no arremesso, existem duas que são críticas para 0 aumento da dor no ombro: a fase de preparação para o arremesso ("cocking") e a fase final de desaceleração ("follow-through"), que são descritas por FERRARRI (1994) ${ }^{10}$. A associação de 
movimentos repetitivos acima da cabeça e erros de treinamento referentes ao gesto esportivo do lançamento, provocam o aparecimento de sintomas e, supostamente, provocam também as acomodações tissulares adaptativas que acarretam a instabilidade glenoumeral. ${ }^{4,6,15,17} \mathrm{Em}$ nosso estudo foi observado que tanto os atletas arremessadores quanto os atletas não arremessadores apresentaram desequilíbrio de força máxima muscular para o grupo dos rotadores externos, quando estes são avaliados em posição de $90^{\circ}$. de abdução. Este predomínio foi descrito pela literatura. ${ }^{7} \mathrm{Em}$ nosso estudo, foi escolhido o dinamômetro isométrico de mola com fixação, por se adequar à disponibilidade do equipamento e pela melhor adequação da posição de avaliação do atleta. A literatura descreve que a margem de erro entre a dinamometria isométrica de mola e a dinamometria isocinética com relação ao membro superior e ao posicionamento do mesmo, para a avaliação, é semelhante. ${ }^{1,5,7,13,19,20,21}$ A literatura descreve que a força dos músculos rotadores mediais do ombro em posição de abdução a $90^{\circ}$. e flexão de $90^{\circ}$. de cotovelo é maior que a dos rotadores laterais. ${ }^{5,8,20}$ No entanto, não encontramos avaliação feita em amostra de atletas com idade semelhantes às da nossa população. A análise de nossa amostra sugere que, apesar de existir um número maior de atletas com predomínio dos grupos musculares rotadores mediais, essa diferença não é significante, achado inesperado, uma vez que imaginávamos que no lado dominante, submetido a constantes sobrecargas, seria natural encontrarmos um predomínio marcante e significativo dos músculos rotadores mediais, tanto em posição neutra, quanto em posição de abdução de $90^{\circ}$.

\section{CONCLUSÃO:}

De acordo com os dados obtidos neste estudo e as variáveis estudadas, podemos concluir que a repetição do gesto de arremesso não mostrou ser o fator determinante para o desequilíbrio de força máxima entre rotadores mediais e laterais, segundo o teste por dinamometria isométrica de mola.

Foi encontrado desequilíbrio significante de força máxima muscular de rotadores laterais no ombro não dominante dos atletas não arremessadores e rotadores medias no ombro não dominante dos atletas arremessadores, ambos em posição neutra de teste $(P=0.001)$ e desequilíbrio significante de força máxima muscular de rotadores medias no ombro não dominante dos atletas não arremessadores e rotadores laterias no ombro não dominante dos atletas arremessadores, ambos em posição de $90^{\circ}$ de abdução de teste $(P<0.001)$.

Não foi identificada a associação entre o desequilíbrio de força máxima muscular e a instabilidade glenumeral.

\section{REFERÊNCIAS BIBLIOGRÁFICAS:}

1. BANKES, M., CROSSMAN, J., EMERY, R.: A Standart Method of Shoulder Strengt Measurement for The Constant Score with a Spring Balance. J. Shoulder Elbow Surg. (7):116- 121, 1998.

2. BIGLIANI, L.U., CODD, T.P., CONNOR, P. M., LEVINE, W. N., LITTLEFIELD, W.N., HERSHON, S.J.: Shoulder Motion and Laxity the Professional Baseball Player. Am J Sports Med 25(5): 609613, 1997. 
3. BISSON, L. J., ANDREWS, J. R. - Classification and Mechanisms of Shoulder Injuries in Throwers. In ANDREWS, R.A.; ZARINS, B; WILK, K. E.: Injuries in Baseball. $1^{\text {a }}$ ed. LippincottRaven Publishers. 1998. p. 47-57.

4. BRASIL, R.F., FILLARDI, C.S.F., MENITTI, E.L., SOUZA, A.S.:Avaliação de Pacientes Operados devido à Síndrome do Pinçamento com ou sem Lesão do Manguito Rotador. Rev Bras Ortop, 30(9): 649-654, 1995.

5. BROWN, L. P., NIEHUES, S. L., HARRAH, A., YAVORSKY, P., HERSHAMAN, H. P.: Upper Extremity range of Motion and Isokinetic Strength of the Internal and External Shoulder Rotators in Major League Baseball Players. Am J Sports Med 16(6): 577-585, 1988.

6. CARAZZATO, J.G., AMBRÓSIO, M. A, MARQUES NETO, J. AZZE, R. J., AMATUZZI, M. M.: Incidência de lesões pregressas do aparelho locomotor encontradas em avaliação global de 271 atletas jovens de elite de dez modalidades esportivas. Revista Bras Ortop. 33(12):919-929. 1998.

7. CARPENTER, J. E., BLAISER, R. B., PELLIZZON, G. G.: The Effects of Muscles Fatigue on Shoulder Joint Position Sense. Am. J. Sport Med. 26(2); 262-265, 1998.

8. CODINE, P., BERNARD, P.L., POCHOLLE, M., BENAIM, C: Influence of sports discipline on shoulder rotator cuff balance. Med Sci Sports Exerc. 29(11):1400-05, 1997.

9. FLEISIG, G. S., ESCAMILLA, R. F., BARRENTINE, S. W. - Biomechanics of Pitching: Mechanism and Motion Analysis. In ANDREWS, R.A.; ZARINS, B; WILK, K. E.: Injuries in Baseball. $1^{\text {a }}$ ed. Lippincott-Raven Publishers. 1998. p. 3-23.

10. FERRARI, J., FERRARI, D., COUSMAS, J.: Posterior Ossification of the Shoulder: The Bennett Lesion-Etiology, Diagnosis and Treatment. Am. J. Sports Med. 22(2):171-175, 1994.

11. FLEISIG, G.S., ANDREWS, J.R., CHARLES, JD, DILMAN, C.J., ESCAMILA, R.F.: Kinetics of baseball pitching with implications about injury mechanisms. Am J Sports Med, 23(2): 233-239, 1995.

12. GOMES, T.: Metodologia do Treinamento Científico. $4^{a}$. ed. Summus Editorial. 1986. p. 25- 183.

13. HUERLEY, J. A, ANDERSON, T. E., DEAR, W., ANDRISH, J. T., BERGELD, J. A, WEIKER, G.G.: Posterior sholuder instability. Am J Sports Med. 20(4):396-400, 1992.

14. IRELAND, M. L., SATTERWHITE, Y.E. - Shoulder Injuries. In ANDREWS, R.A.; ZARINS, B; WILK, K. E.: Injuries in Baseball. $1^{a}$ ed. Lippincott-Raven Publishers. 1998. P. 271-282.

15. MAESENERR, M., JAOVISIDHA, S., JACOBSON, J. A, TAM, W., SCHILS, J. P., SARTORIS, D. J., FRONEK, J. RESNICK, D.: The Bennett lesion of the shoulder. J Comp Assis Tomo. 22(1):3134, 1998.

16. MAGILL, R. A.: Aprendizagem Motora: Conceitos e aplicações. $1^{\text {a }}$ ed. Editora Edgard Blücher Ltda. 1986. p.159-169.

17. MONTORO, M: Prevenção das Lesões do Manguito Rotador em Atletas Fisiculturistas em "Off Season". Fisioterapia em Movimento. 4(2); 9-27, 1994.

18. SIEGEL, S.: Estatística não paramétrica (para as ciências do comportamento). $1^{\text {a }}$ ed. Editora Mc Graw-Hill Itda. 1975. 350p.

19. SIROTA, S.C., MALANGA, G.A., EISCHEN, J.J., LASKOWSKI, E.R.: An Eccentric and Concentric Strength Profile of Shoulder External and Internal Rotator Muscles in Professional Baseball Pitchers. Am J Sports Med 25(1): 59- 64, 1997.

20. WILK, K.E., ANDREWS, J.R., ARRIGO, C.A., KEIRNS, M.A., ERBER: The Strength Characteristics of Internal and External Rotator Muscles in Professional Baseball Pitchers. Am J Sports Med 21(1): 61-66, 1993. 
21. WINDT, D. A W. M., HEIJDEN, G. J. M. G., WINTER, A F., KOES, B. W., DEVILLÉ, W., BOUTER, L. M.: The responsiveness of the shoulder disability questionnaire. Ann Rheum Dis 57:82-87, 1998. 
São Paulo, 05 de Maio de 2003.

A/C Centro de Docência e Pesquisa do Curso de Fisioterapia da Faculdade de Medicina da USP.

Venho por meio desta solicitar a apreciação do artigo que se refere ao resumo de minha tese de mestrado pela Universidade Federal de São Paulo/Escola Paulista de Medicina.

Agradeço pela oportunidade desta apreciação e aguardo retorno.

Ana Cristina de Sousa 